\title{
Density of $M$-sets in arithmetic progression
}

\author{
by
}

\section{Soma Gupta and Amitabha Tripathi (New Delhi)}

Following Motzkin, for a given set $M$ of positive integers, a set $S$ of nonnegative integers is called an $M$-set if $a, b \in S$ implies $a-b \notin M$. In an unpublished problem collection, Motzkin posed the problem of determining the quantity

$$
\mu(M)=\sup _{S} \bar{\delta}(S)
$$

where the supremum is taken over the class of all $M$-sets $S$. As usual, the upper density of $S, \bar{\delta}(S)$, is defined by $\lim \sup _{n \rightarrow \infty} S(n) / n$, where $S(x)$ denotes the number of elements in $S$ less than or equal to $x$.

Cantor and Gordon [1] solved the problem for the cases where $M$ has at most two elements besides obtaining partial results for the general case. Haralambis [2], besides giving some general estimates, determined $\mu(M)$ for most members of the families $\{1, j, k\}$ and $\{1,2, j, k\}$. In this note, we determine $\mu(M)$ in the case where the elements of $M$ are in arithmetic progression.

We recall the following results proved by Cantor and Gordon [1]:

Lemma A. If $M_{1}=\left\{m_{1}, m_{2}, \ldots\right\}$ and $M_{2}=\left\{d m_{1}, d m_{2}, \ldots\right\}$, where $d$ is a positive integer, then $\mu\left(M_{1}\right)=\mu\left(M_{2}\right)$.

Lemma B. Let $M=\left\{m_{1}, m_{2}, \ldots\right\}$ and let $c$ and $m$ be positive, relatively prime integers with

$$
d=\min _{k}\left|c m_{k}\right|_{m}
$$

where $|x|_{m}$ denotes the absolute value of the absolutely least remainder of $x$ $(\bmod m)$. Then $\mu(M) \geq d / m$.

This implies that for any set $M=\left\{m_{1}, m_{2}, \ldots\right\}$,

$$
\mu(M) \geq \sup _{\operatorname{gcd}(c, m)=1} \frac{1}{m} \min _{k}\left|c m_{k}\right|_{m} .
$$

1991 Mathematics Subject Classification: Primary 11B05. 
Lemma C. Let $M$ be a given set of positive integers, $\alpha \in[0,1]$, and suppose that for any $M$-set $S$ with $0 \in S$ there exists a positive integer $k$ (possibly dependent on $S$ ) such that $S(k) \leq(k+1) \alpha$. Then $\mu(M) \leq \alpha$.

TheOREM. If $M=\{a, a+d, a+2 d, \ldots, a+(n-1) d\}$ with $\operatorname{gcd}(a, d)=1$ and $n \geq 1$, then

$$
\mu(M)= \begin{cases}\frac{2 a+(n-1)(d-1)}{2\{2 a+(n-1) d\}} & \text { if } d \text { is odd } \\ \frac{1}{2} & \text { if } d \text { is even } .\end{cases}
$$

Pr o o f. By Lemma A, it suffices to consider the case where $\operatorname{gcd}(a, d)=1$. If $d$ is even, then $\{1,3,5, \ldots\}$ is an $M$-set, and $\mu(M)=1 / 2$.

Suppose now that $d$ is odd. If we write $m$ for $2 a+(n-1) d$, then $\operatorname{gcd}(d, m)=1$, and so $d x_{0} \equiv 1(\bmod m)$ for some integer $x_{0}$. Let $d x_{0}=$ $1+m q$. Then $x_{0}$ and $m q$, and hence $x_{0}$ and $(n-1) q$, are of opposite parity, and so

$$
\begin{aligned}
a x_{0} & =\frac{m-(n-1) d}{2} x_{0} \\
& =\frac{m\left\{x_{0}-(n-1) q\right\}-(n-1)}{2} \equiv \frac{m-(n-1)}{2}(\bmod m) .
\end{aligned}
$$

Therefore, for $0 \leq k \leq n-1$,

$$
(a+k d) x_{0} \equiv \frac{m}{2}+\left(k-\frac{n-1}{2}\right)(\bmod m),
$$

and by Lemma B, we have

$$
\mu(M) \geq \frac{2 a+(n-1)(d-1)}{2\{2 a+(n-1) d\}} .
$$
let

Conversely, let $S$ be any $M$-set with $0 \in S$, and for $m=2 a+(n-1) d$

$$
\bigcup_{i=1}^{(m-n-1) / 2} A_{i} \cup B
$$

be a partition of $\{0,1, \ldots, m-1\}$, where $B=\{0, a, a+d, \ldots, a+(n-1) d\}$ and $A_{i}=\{i d, a+(n+i-1) d\}, 1 \leq i \leq(m-n-1) / 2$, the elements of $A_{i}$ taken modulo $m$. Hence, $|S \cap B|=1$ and $\left|S \cap A_{i}\right| \leq 1$ for each $i$.

Therefore,

$$
S(m-1) \leq 1+\frac{m-(n+1)}{2}=\frac{2 a+(n-1)(d-1)}{2}
$$

for any $M$-set $S$. 
Thus, by Lemma C, $\mu(M) \leq\{2 a+(n-1)(d-1)\} /(2 m)$. Therefore,

$$
\mu(M)=\frac{2 a+(n-1)(d-1)}{2\{2 a+(n-1) d\}} .
$$

We observe that the results of Cantor and Gordon [1] for $\mu(M)$ when $|M| \leq 2$ follow easily from this theorem. Also, as $n \rightarrow \infty, \mu(M) \rightarrow$ $(d-1) /(2 d)$ if $d$ is odd and to $1 / 2$ if $d$ is even.

\section{References}

[1] D. G. Cantor and B. Gordon, Sequences of integers with missing differences, J. Combin. Theory Ser. A 14 (1973), 281-287.

[2] N. M. Haralambis, Sets of integers with missing differences, ibid. 23 (1977), 22-33.

Department of Mathematics

Indian Institute of Technology

Hauz Khas

New Delhi 110 016, India

E-mail: atripath@maths.iitd.ernet.in 